\title{
Long noncoding RNAs show differential expression profiles and display ceRNA potential in cholesteatoma pathogenesis
}

\author{
JUANJUAN GAO ${ }^{1,3}$, QI TANG ${ }^{1,3}$, XIAOHUI ZHU ${ }^{1,3}$, SHIHUA WANG $^{2}$, YONGLI ZHANG ${ }^{1,3}$, \\ WENBIN LIU ${ }^{1,3}$, ZHIQIANG GAO ${ }^{1,3}$ and $\mathrm{HUA} \mathrm{YANG}^{1,3}$ \\ ${ }^{1}$ Department of Otolaryngology, Peking Union Medical College Hospital; ${ }^{2}$ Center of Excellence in Tissue Engineering, \\ Key Laboratory of Beijing, Institute of Basic Medical Sciences and School of Basic Medicine; \\ ${ }^{3}$ Translational Medicine Center, Peking Union Medical College Hospital, Chinese Academy \\ of Medical Sciences and Peking Union Medical College, Beijing 100730, P.R. China
}

Received November 1, 2017; Accepted March 16, 2018

DOI: $10.3892 /$ or.2018.6320

\begin{abstract}
Cholesteatoma is a pathologically benign but clinically destructive middle ear disease, which is caused by excessive epidermal migration and uncontrolled hyperproliferation of keratinocytes of squamous epithelium, leading to various clinical manifestations and serious complications, such as hearing loss, dizziness, facial paralysis, meningitis, and hydrocephalus. However, the pathogenesis of cholesteatoma is still not fully understood. Herein, we performed microarray analysis to identify the differentially expressed patterns of IncRNAs in cholesteatoma for the first time. Our data indicated that compared with matched normal skin tissue, IncRNA expression profiles were significantly altered in cholesteatoma. A total of 787 lncRNAs were identified (fold change $\geq 2.0, \mathrm{P}<0.05$ ), consisting of 181 upregulated and 606 downregulated lncRNAs. Furthermore, by constructing an lncRNA/miRNA/mRNA competing endogenous RNA (ceRNA) network, we found that lncRNAs, such as IncRNA-uc001kfc.1, had ceRNA potential in cholesteatoma formation. In conclusion, lncRNAs were aberrantly expressed in cholesteatoma compared with normal skin tissues and may play important roles in cholesteatoma formation. Our findings shed novel light on the molecular mechanism of cholesteatoma pathogenesis and suggest that lncRNAs may be potential therapeutic targets for cholesteatoma.
\end{abstract}

Correspondence to: Dr Hua Yang, Department of Otolaryngology, Peking Union Medical College Hospital, Chinese Academy of Medical Sciences and Peking Union Medical College, Beijing 100730, P.R. China

E-mail: yanghua702@163.com

Key words: cholesteatoma, long non-coding RNA (lncRNA), microarray analysis, competing endogenous RNA (ceRNA), network

\section{Introduction}

Cholesteatoma is a pathologically benign chronic middle ear disease, but potentially destructive epithelial lesion caused by aberrant keratinocyte proliferation and migration which can result in erosion of adjacent osseous structures, leading to various clinical manifestations and serious complications (1). Bone resorption of the middle and inner ear can cause hearing loss, tinnitus, dizziness, and facial paralysis. Erosion of the tegmen may lead to many severe intracranial complications, including sigmoid sinus thrombophlebitis, extradural, subdural or brain abscess, meningitis, and hydrocephalus (2-5). To date, there is no drug therapy for cholesteatoma and the standard treatment is surgical resection (2). In addition, postoperative recurrences are very common; thus many patients undergo multiple surgeries $(4,5)$.

Nevertheless, the exact underlying cellular and molecular mechanisms of cholesteatoma are still unclear. Previous studies indicated that, during cholesteatoma formation, epithelial proliferation and keratinocyte differentiation are regulated by high activity of growth factors (6). Cytokines are also supposed to play important roles in the aggressive behavior of cholesteatoma (6). In addition to those inflammatory mediators and growth factors, aberrant expression of regulatory microRNAs (miRNAs), the best-studied non-coding RNAs (ncRNAs), play a role in cholesteatoma formation (7-10). However, due to a complementarity of merely 6 base pairs (bp) nt can be sufficient for target recognition, a miRNA can regulate hundreds of mRNAs, and correspondingly, a mRNA can be regulated by several miRNAs (11), leading to the difficulties encountered when clarifying the molecular mechanisms underlying cholesteatoma.

In 2011, Salmena et al introduced the competitive endogenous RNA (ceRNA) hypothesis that all types of RNA transcripts can communicate with and regulate each other by using shared microRNA response elements (MREs) (12), which provides us with a new perspective for research on the cholesteatoma formation process. CeRNAs, also termed endogenous miRNA sponges, act as decoy molecules that absorb active miRNAs and buffer regulation activities of miRNAs on their targeted mRNAs (13). 
Salmena et al proposed that the ceRNA protagonists are miRNAs, the protein coding genes, pseudogenes, and long non-coding RNAs (lncRNAs). IncRNAs, which are noncoding RNAs longer than $200 \mathrm{nt}$, can regulate each other with miRNAs and function in almost every aspect of human biology, including chromatin modification, transcriptional regulation, post-transcriptional processing, RNA editing, RNA trafficking, cell cycle regulation, alternative splicing, and organelle biogenesis $(14,15)$. In the past decade, with the emergence of microarray and high-throughput sequencing techniques, IncRNAs have attracted the attention and have been involved in epigenetic mechanisms of various diseases and display ceRNA potential in many diseases (16-23). Nevertheless, to date, no studies have addressed the expression profiles of IncRNAs in cholesteatoma.

Therefore, in the present study, we performed microarray analysis to identify the differentially expressed patterns of IncRNAs and mRNAs between cholesteatoma and normal skin tissues. Quantitative RT-PCR was applied to confirm the reliability of microarray expression data. With specific bioinformatics approaches, we constructed the IncRNA-miRNA-mRNA ceRNA network to explore the ceRNA potential of IncRNAs in cholesteatoma.

\section{Material and methods}

Patients and specimens. Cholesteatoma tissue and matched post-auricular normal skin tissue were sampled in each patient. A total of 7 patients aged 18-32 years who underwent unilateral middle ear cholesteatoma surgeries between June 2016 and November 2016 at the Department of Otorhinolaryngology, Peking Union Medical College Hospital were enrolled in this study. All participants provided written informed consent and were clinically and histologically confirmed to present with cholesteatoma. All specimens were stored at $-80^{\circ} \mathrm{C}$ immediately after collection for later RNA extraction. Ethical approval for the study was obtained from the Ethics Committee of Peking Union Medical College Hospital (no. S-K292).

RNA extraction and quality control. Total RNA was extracted from cholesteatoma and post-auricular skin tissues using TRIzol reagent (Invitrogen, Carlsbad, CA, USA), according to the manufacturer's instructions. RNA quantity and quality were assessed by NanoDrop ND-1000. RNA integrity was assessed by standard denaturing agarose gel electrophoresis or Agilent 2100 Bioanalyzer (Agilent Technologies, Santa Clara, CA, USA).

Microarray analysis. Four pairs of cholesteatoma and post-auricular skin tissues were used for microarray assay to determine differentially expressed lncRNAs and mRNAs comparing cholesteatoma and post-auricular skin specimens. Sample labeling and array hybridization were performed according to the Agilent One-Color Microarray-Based Gene Expression Analysis protocol (Agilent Technologies, Englewood, CO, USA) with minor modifications. The hybridized arrays were washed, fixed, and scanned using the Agilent DNA Microarray Scanner (part no. G2505C). Agilent Feature Extraction software (version 11.0.1.1) was used to analyze acquired array images. Quantile normalization and subsequent data processing were performed using the GeneSpring GX v12.1 software package (Agilent Technologies, Englewood, CO, USA). After quantile normalization of the raw data, lncRNAs and mRNAs in the 8 samples flagged as 'Present' or 'Marginal' were chosen for further data analysis. Differentially expressed lncRNAs and mRNAs with statistical significance between the two groups were identified through P-value/FDR filtering. Differentially expressed lncRNAs and mRNAs between the two samples were identified through Fold Change filtering. Pathway analysis and Gene ontology (GO) analysis were applied to determine the roles played by these differentially expressed mRNAs in these biological pathways or GO terms. Hierarchical clustering and combined analysis were performed by using in-house scripts.

Functional group analysis. GO provides a controlled vocabulary to describe gene function and relationships between these concepts in any organism (http://www.geneontology.org). GO covers three aspects: Biological process, cellular component, and molecular function. Fisher's exact test was applied to determine whether the overlap between the differentially expressed (DE) list and the GO annotation list is greater than that expected by chance. The $-\log _{10}$ (P-value) was used to denote the significance of the GO term enrichment in the DE genes. FDR represents the false discovery rate. The lower the P-value is, the more significant the GO term is (a P-value $<0.05$ is recommended). Pathway analysis was performed to collect pathway clusters on the molecular interaction and reaction networks by mapping genes to the Kyoto Encyclopedia of Genes and Genomes (KEGG) pathways (http://www.genome.jp/kegg/). The $-\log _{10}$ (P-value) denotes the significance of the pathway correlations. The lower the P-value is, the more significant the correlation is (a P-value $<0.05$ is recommended).

Quantitative real-time PCR validation. The selected $\operatorname{lncRNAs}$ and primers used for qRT-PCR were designed using Primer 5.0 and synthesized by Generay Biotech (Shanghai, China). $\beta$-actin was used as an internal control for all samples. Primer sequences were as follows: $\beta$-actin forward, 5'-GTGGCCGAG GACTTTGATTG-3' and reverse, 5'-CCTGTAACAACGCAT CTCATATT-3'; ENST00000415386 forward, 5'-TGGAGT AGGCACAGACGGAA-3' and reverse, 5'-GACTGTGGT ACAATCGCTTCG-3'; ENST00000420253 forward, 5'-CGA TGTCAACCCTCGAACCT-3' and reverse, 5'-TCAGATGTG GCCCACTGTCT-3'; NR_024468 forward, 5'-GCTTACATT TTCTCTGCCATCTC-3' and reverse, 5'-CCACTTTCCTTT СТCTCTCTTCT-3'; T044224 forward, 5'-TGCGAAAAC ACTGCGATTG-3' and reverse, 5'-GGTTGGACCAGACCA GATGA-3'; T347175 forward, 5'-GAAAGAGGCACTGCT GTTGA-3' and reverse, 5'-GGCTGCTCCCAGAATAGA TAG-3'; uc001kfc.1 forward, 5'-TTCCCAGAAGGCGTA GGTT-3' and reverse, 5'-GACAGAGTCTCCCTCTATCAT CC-3'; qRT-PCR was performed by using ViiA 7 Real-time PCR System (Applied Biosystems, San Jose, CA, USA) with a SYBR expression assay system (Takara Biotechnology, Co., Ltd., Dalian, China). The PCR reaction conditions were as follows: An initial denaturation at $95^{\circ} \mathrm{C}$ for $10 \mathrm{~min}$, followed by $40 \mathrm{PCR}$ cycles at $95^{\circ} \mathrm{C}$ for $10 \mathrm{sec}$ and $60^{\circ} \mathrm{C}$ for $60 \mathrm{sec}$. Then annealing and extension at $95^{\circ} \mathrm{C}$ for $10 \mathrm{sec}, 60^{\circ} \mathrm{C}$ for $60 \mathrm{sec}$ 
and $95^{\circ} \mathrm{C}$ for $15 \mathrm{sec}$. Each sample was assayed in triplicates. The $2^{-\Delta \Delta \mathrm{Ct}}$ method was used to determine fold-change in gene expression in the cholesteatoma samples relative to the normal skin samples. For statistical analysis, we used unpaired t-test to compare the expression of lncRNAs between cholesteatoma and normal skin samples. $\mathrm{P}<0.05$ was considered to indicate a statistically significant difference.

Competing endogenous network analysis. All potential miRNA response elements were searched based on the sequences of lncRNAs and mRNAs. LncRNA/miRNA/mRNA interactions were predicted by the overlapping of the miRNA seed sequence binding site both on the chosen dysregulated lncRNAs and the significantly dysregulated mRNA. miRBase V19 was selected to interact with the lncRNAs and mRNAs (http://www.mirbase.org/). LncRNA/miRNA interactions were predicted by miRcode (http://www.mircode.org/). miRNA/mRNA interactions were predicted by miRanda(www. microrna.org/) and TargetScan (http://www.targetscan.org/).

\section{Results}

LncRNAs and mRNAs present significantly different expression profiles in cholesteatoma compared to matched normal skin specimens. A microarray analysis was performed to profile differences in IncRNA and mRNA expression between 4 pairs of cholesteatoma and matched normal skin samples. According to microarray expression profiling data, 11,815 lncRNAs and 7,692 mRNAs were detected. The lncRNAs were carefully collected from the most authoritative databases such as RefSeq, UCSC Known Genes, Gencode, and other related sources. According to their relation with protein-coding genes, all deregulated lncRNAs in cholesteatoma were classified into six categories: Bidirectional (4.46\%), exon sense-overlapping (1.16\%), intron sense-overlapping $(3.47 \%)$, natural antisense $(8.75 \%)$, intronic antisense $(12.71 \%)$ and intergenic $(69.47 \%)$ (Fig. 1). Hierarchical clustering revealed that IncRNA and mRNA expression patterns between cholesteatoma and matched normal skin tissues were distinguishable (Fig. 2A and B). Volcano plots were used for visualization and assessment of the variation (or reproducibility) of IncRNA and mRNA expression between cholesteatoma and matched normal skin tissues (Fig. 2C and D).

Furthermore, by setting a threshold for differential expression at changes $\geq 2$.0-fold, we identified 787 lncRNAs and 591 mRNAs that were differentially expressed $(\mathrm{P}<0.05)$ between cholesteatoma and matched normal skin tissues (Fig. 2C and D). Among them, in cholesteatoma samples, $181 \mathrm{lncRNAs}$ and 155 mRNAs were upregulated (fold change $\geq 2.0, \mathrm{P}<0.05$ ) and $606 \mathrm{lncRNAs}$ and 436 mRNAs were downregulated (fold change $\geq 2.0, \mathrm{P}<0.05$ ) compared with normal skin samples (Fig. 2C and D). The microarray profile and RNAseq data sets have been deposited into Gene Expression Omnibus (GEO) with accession number GSE102673 (https://www.ncbi.nlm.nih.gov/geo/query/acc. cgi?acc $=$ GSE102673).

GO analyses display cholesteatoma-related gene functions of differentially expressed mRNAs. GO analysis was performed to determine the gene and gene product enrichment in
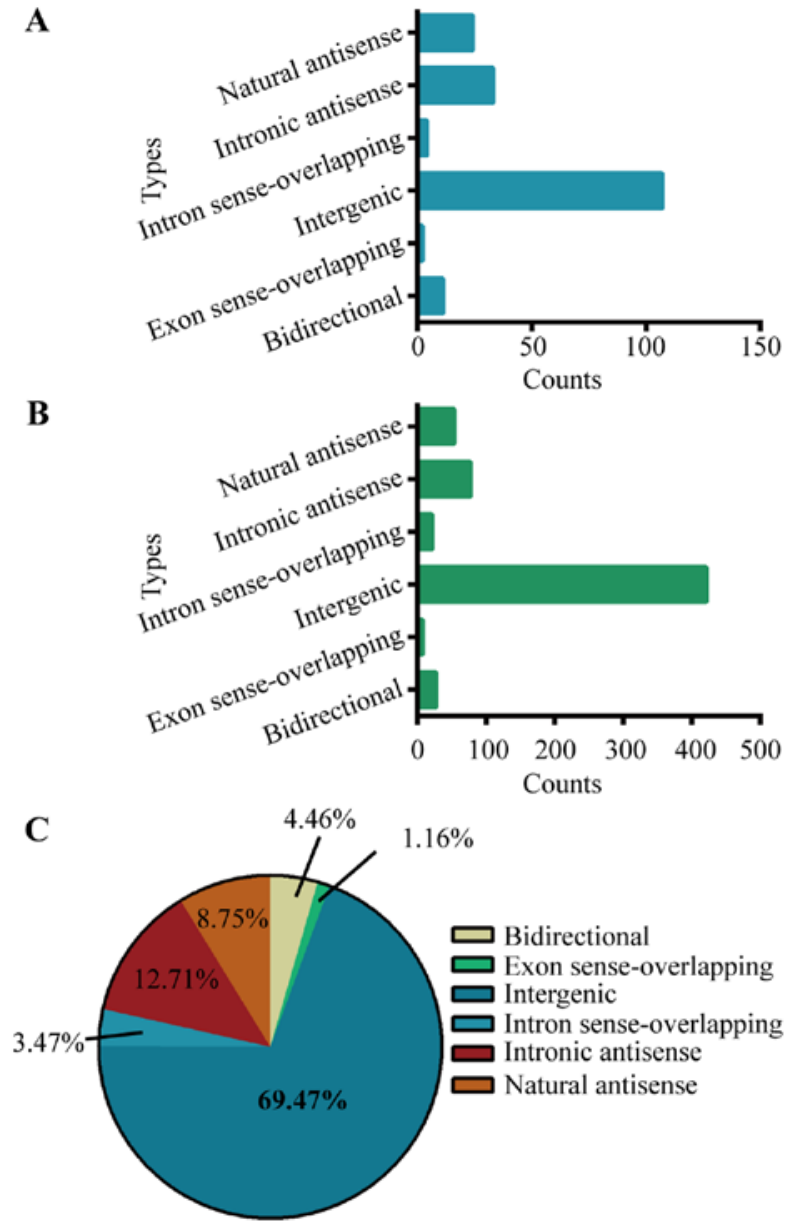

Figure 1. Counts and types of differentially regulated lncRNAs. (A) Counts and types of upregulated lncRNAs. (B) Counts and types of downregulated lncRNAs. (C) All dysregulated lncRNAs were classifed into six categories: Bidirectional (4.46\%), exon sense-overlapping (1.16\%), intron sense-overlapping $(3.47 \%)$, natural antisense $(8.75 \%)$, intronic antisense $(12.71 \%)$ and intergenic $(69.47 \%)$.

categories including biological process (BP), molecular function (MF), and cellular component (CC). LncRNAs can regulate the neighboring and overlapping coding gene expression. Thus, GO enrichment analysis of differentially expressed mRNAs may partially display the role of differentially regulated lncRNAs. In the present study, the target genes of deregulated mRNAs were analyzed and compared with those in cholesteatoma. In the GO analysis, by setting $\mathrm{P}<0.05$, the upregulated and downregulated mRNAs were analyzed separately and the top-10 enriched GO terms of $\mathrm{BP}, \mathrm{CC}, \mathrm{MF}$ were listed (Fig. 3). In BP analysis, regulation of phosphatidylinositol 3-kinase signaling (GO:0014066), phosphatidylinositol 3-kinase signaling (GO:0014065), and positive regulation of phosphatidylinositol 3-kinase signaling (GO:0014068) belonged to top-10 most enriched processes associated with the upregulated mRNAs. Moreover, phosphatidylinositol 3-kinase binding (GO:0043548) was the most significantly enriched function of the upregulated mRNAs in MF analysis (Fig. 3C). It is notable, since phosphatidylinositol 3-kinase (PI3K) has been reported to play a crucial role in the formation of cholesteatoma (24). Furthermore, growth factor activity (GO:0008083), one of the most significantly enriched 
$\mathbf{A}$
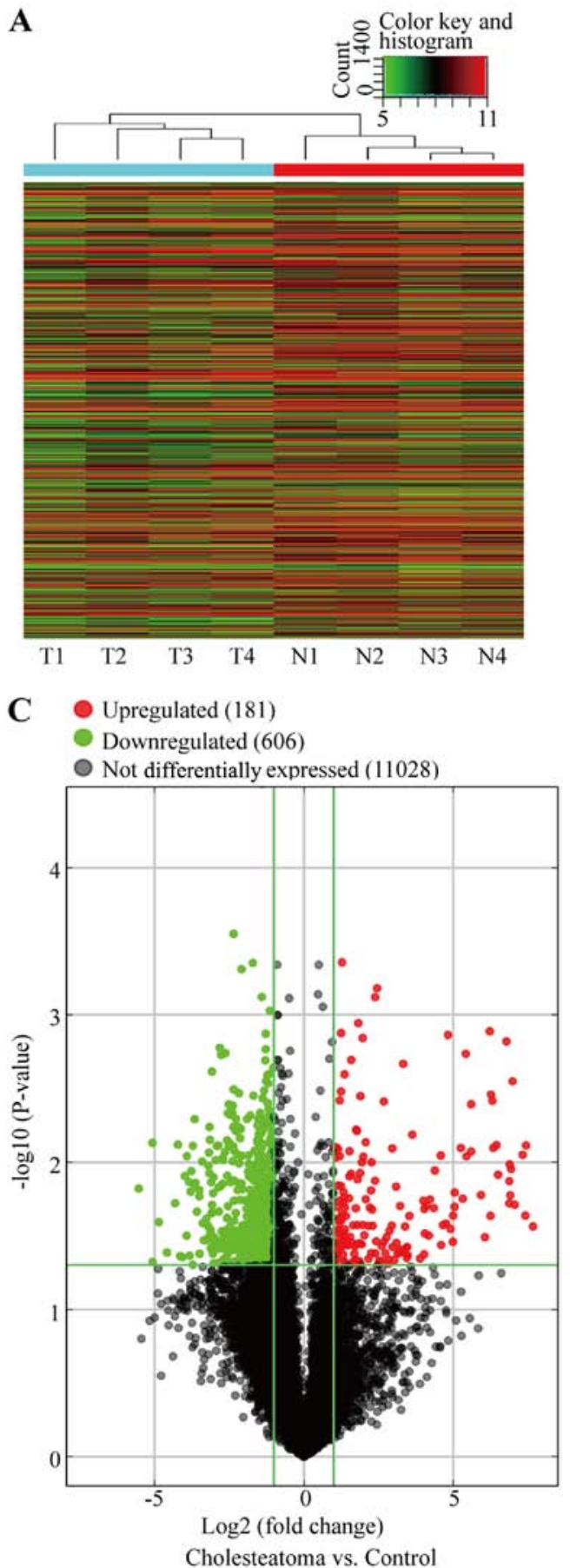

B

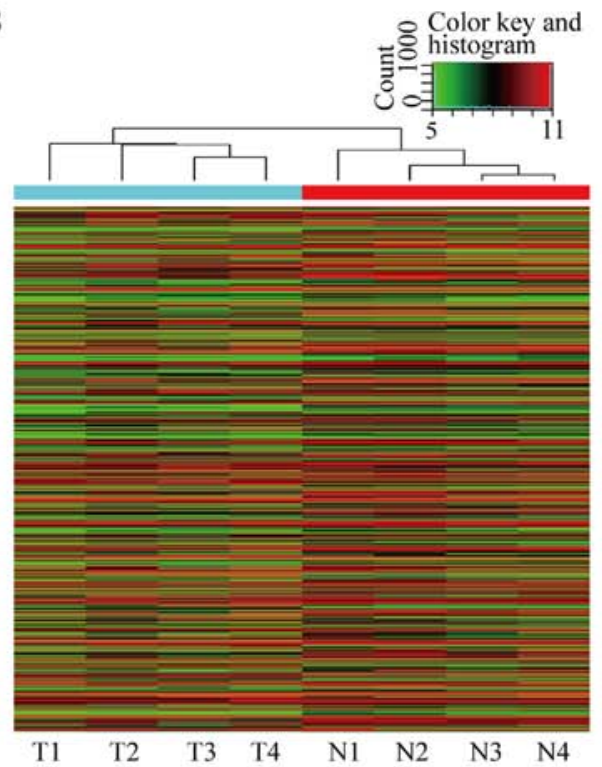

D U Upregulated (155)

- Downregulated (436)

- Not differentially expressed (7101)

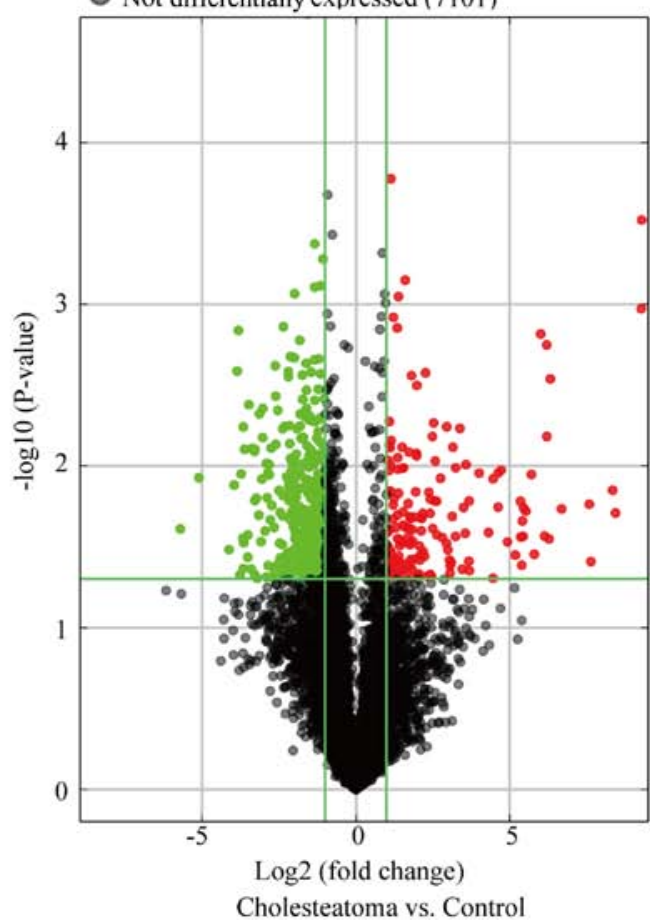

Figure 2. Hierarchical clustering and volcano plots. Hierarchical clustering shows (A) lncRNA and (B) mRNA expression patterns between cholesteatoma (T) and matched normal skin tissues (N). The analysis distinguishes cholesteatomas from matched normal skin tissues by expression level. 'Red' indicates high relative expression, and 'green' indicates low relative expression. Volcano plots were used to visualize and assess the variation (or reproducibility) of (C) lncRNA and (D) mRNA expression between cholesteatoma and matched normal skin tissues. The values of the $\mathrm{x}$ - and y-axes indicate the averaged normalized signal values of the group ( $\log _{2}$ scaled). The green lines indicate fold-change lines.

processes associated with the downregulated mRNAs in MF, is also involved in cholesteatoma formation (25).

Pathway analyses reveal significant enrichment of potential cholesteatoma-related pathways. By mapping genes to KEGG pathways, we performed pathway analysis (http://www .genome.jp/kegg/pathway.html). KEGG pathway enrichment analysis for differentially expressed mRNAs is devised to comprehend pathways and molecular interactions related to genes. Pathway analysis indicated that 10 enriched pathways corresponded to downregulated mRNAs and 7 pathways corresponded to upregulated mRNAs $(\mathrm{P}<0.05$, Fig. 4$)$. The pathways enriched with upregulated lncRNAs were involved in a category 'bacterial invasion of epithelial cells (hsa05100)' that is involved in the molecular mechanisms of cholesteatoma (26). Furthermore, 'viral myocarditis (hsa05416)' and 'Hepatitis B (hsa05161)' were also related to the molecular biology of cholesteatoma (6). 
A

Positive regulation of signaling Carboxylic acid transmembrane transport Regulation of intracellular signal transduction

Regulation of signal transduction Positive regulation of $\mathrm{PI} 3 \mathrm{~K}$ signaling PI3K signaling

Post-Golgi vesicle-mediated transport Regulation of PI3K signaling Golgi to plasma membrane transport Vesicle-mediated transport to the plasma membrane

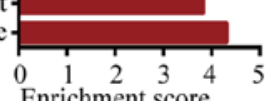

B

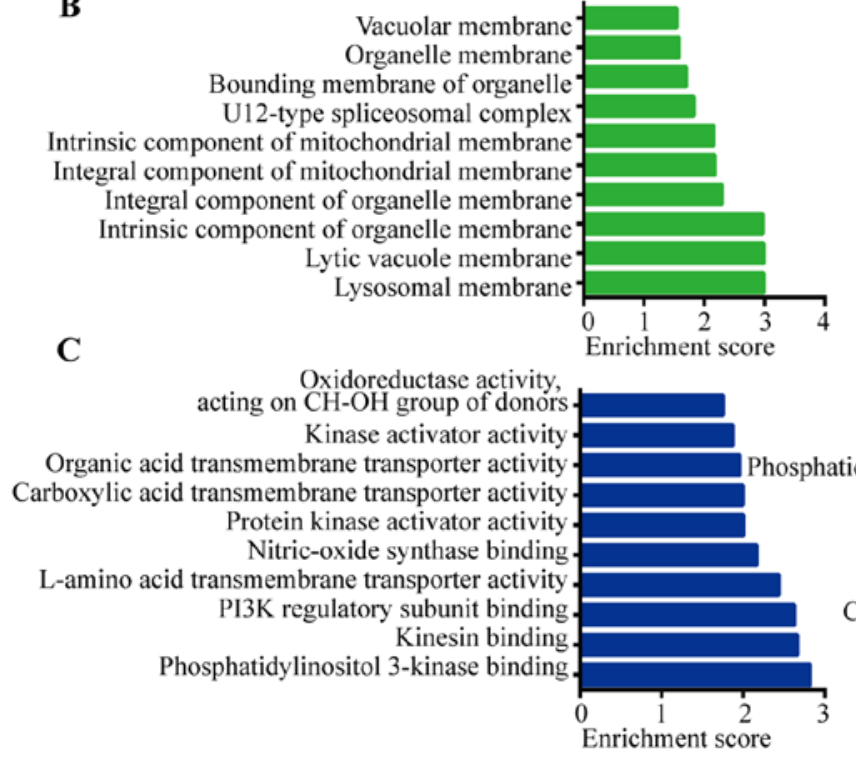

D

Epithelial cell maturation Phenol-containing compound metabolic process Collecting duct development Cerebral cortex radially oriented cell migration Positive regulation of protein targeting to membrane Telencephalon glial cell migration

Cerebral cortex radial glia guided migration Actin cytoskeleton reorganization Layer formation in cerebral cortex

$\mathbf{E}$

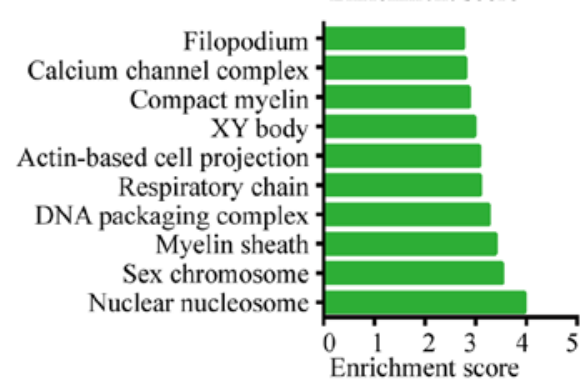

$\mathbf{F}$

Cyclin-dependent protein serine/threonine

kinase inhibitor activity Growth factor activity Ephrin receptor binding
Ephositol 3-kinase regulatory subunit binding Protein-lipid complex binding Lipoprotein particle binding Molecular function regulator Calcium ion transmembrane transporter activity Enzyme activator activity Beta-tubulin binding

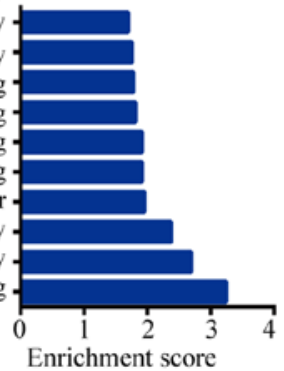

Figure 3. Gene ontology (GO) analysis. The top 10 enrichment score GO terms which corresponded to coding gene functions of (A-C) upregulated and (D-F) downregulated differentially expressed lncRNAs are listed respectively. (A and D) biological process; (B and E) cellular component; (C and F) molecular function. Enrichment score indicates the enrichment score value of the GO ID, equivalent to - $\log _{10}(\mathrm{P}-\mathrm{value})$.
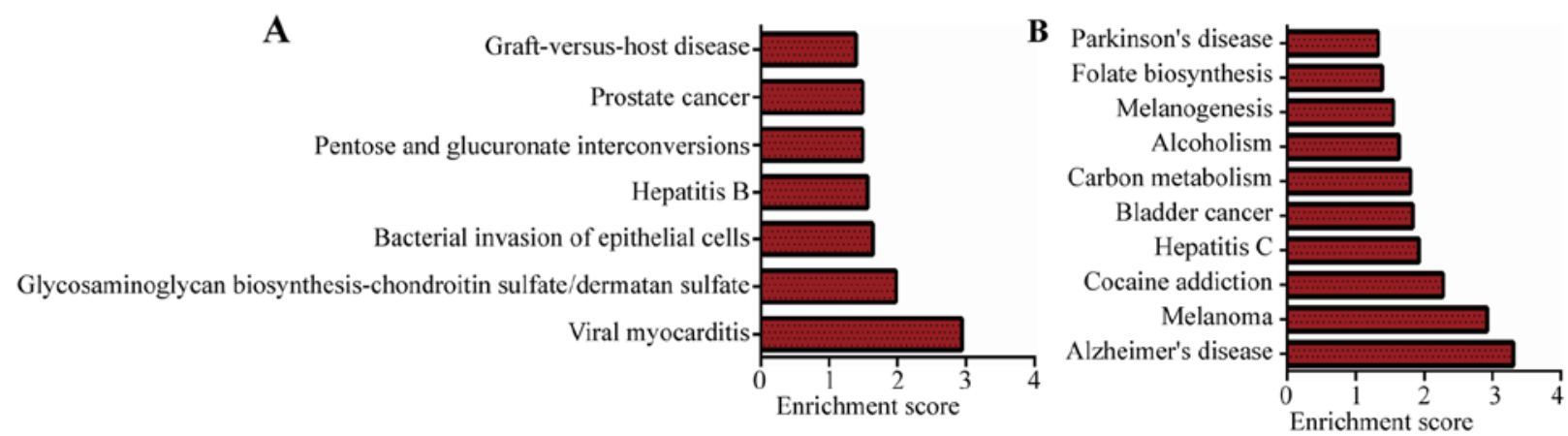

Figure 4. Pathway analysis. The top 10 enrichment score pathways which corresponded to (A) upregulated and (B) downregulated differentially expressed mRNAs are listed respectively. Enrichment score indicates the enrichment score value of the GO ID, equivalent to - $\log _{10}$ (P-value).

Quantitative real-time PCR confirms microarray expression results. Using qRT-PCR, 3 upregulated and 3 downregulated lncRNAs with fold-changes $>2.0$ were randomly selected to verify the microarray data in 7 pairs of samples (4 pairs of original tissues and 3 other pairs of cholesteatoma and normal skin tissues). The qRT-PCR results and microarray data were consistent (Fig. 5), demonstrating that the microarray expression results are highly reliable.

CeRNA network analysis indicates that lncRNAs have ceRNA potential in the pathogenesis of cholesteatoma. CeRNAs are involved in a regulatory mechanism between non-coding RNA and coding RNA based on shared MREs (12). According to the ceRNA hypothesis, ceRNA members can compete for the same MREs to regulate each other. To explore whether lncRNAs have ceRNA potential in the pathogenesis of cholesteatoma, in the present study, we constructed a ceRNA network in cholesteatoma based on the microarray data. We selected 5 significantly differentially expressed lncRNAs (fold change $>2.0, \mathrm{P}<0.05$, Table I), which shared common binding MREs with each other, to constitute an lncRNA/miRNA/mRNA ceRNA network (available upon request). The network was found to be composed of $20 \mathrm{lncRNA}$ nodes, 399 miRNA nodes, and 137 mRNA nodes. In the 

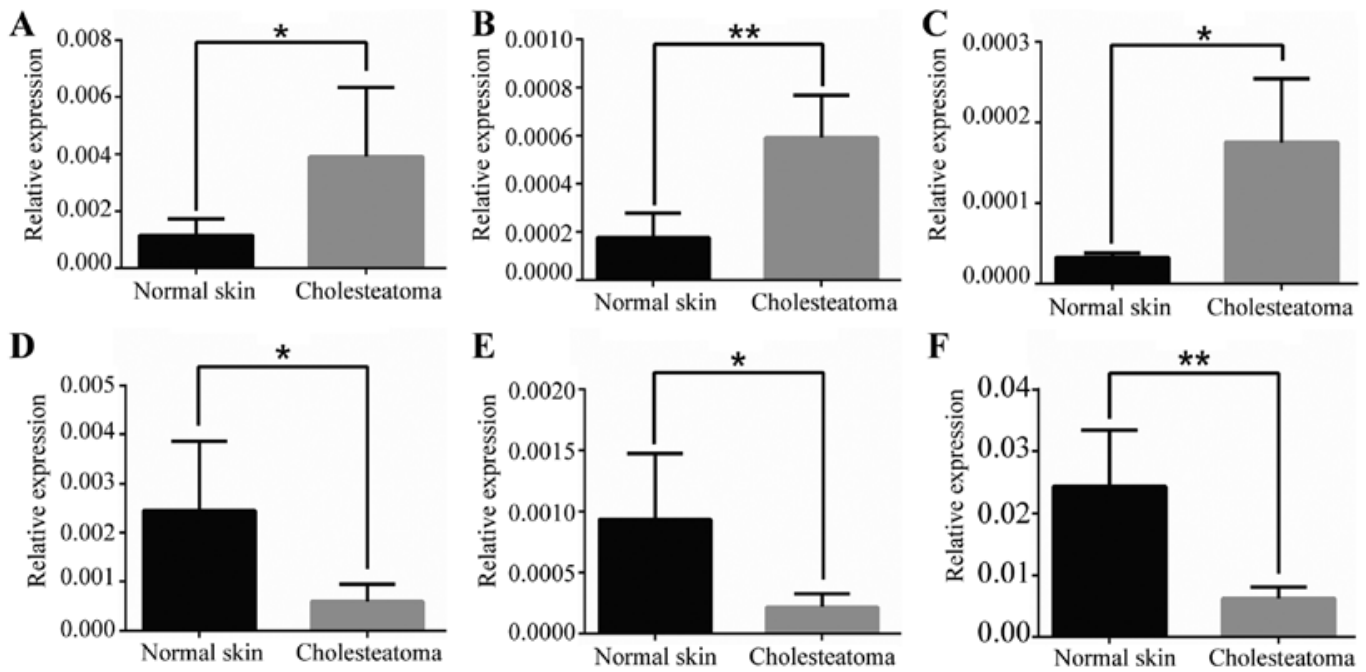

Figure 5. Validation of the microarray data by quantitative RT-PCR. The relative expression levels of 6 deregulated lncRNAs are displayed compared to normal skin and cholesteatoma specimens. (A) ENST00000415386; (B) ENST00000420253; (C) NR_024468; (D) T044224; (E) T347175; and (F) uc001kfc.1. Among them, A, B, and C were upregulated lncRNAs and D, E, and F were downregulated lncRNAs in cholesteatoma. Data are presented as the mean \pm SD; " $\mathrm{P}<0.05$, ${ }^{* *} \mathrm{P}<0.01$, unpaired t-test.

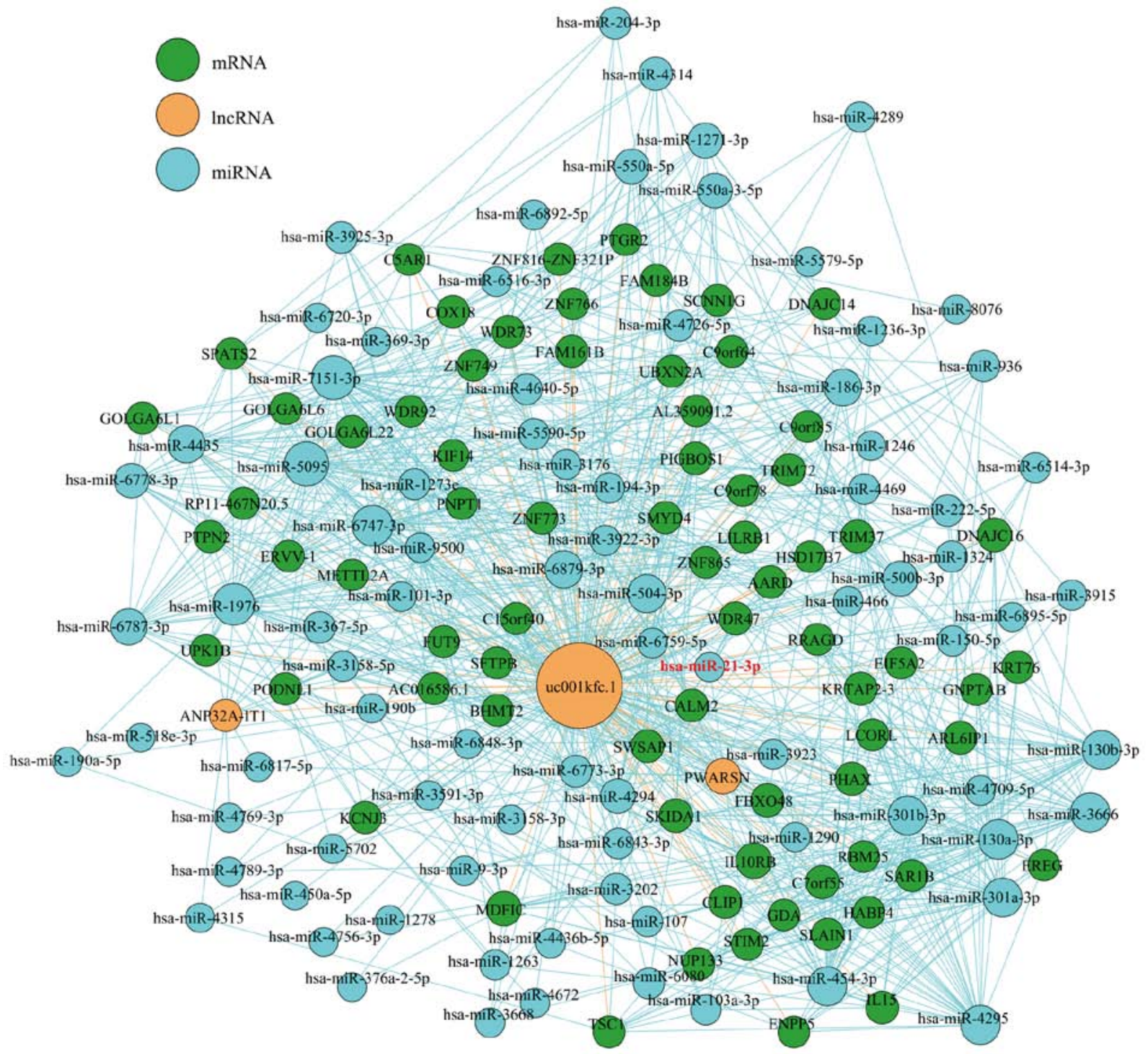

Figure 6. LncRNA-uc001kfc.1 mediates the competing endogenous RNA network. The network was based on lncRNA/miRNA/mRNA interactions. In the network, hsa-miR-21-3p, which shares a microRNA response element (MRE) with uc001kfc.1, was ascertained to play a vital role in cholesteatoma and is highlighted in red font (interaction with uc001kfc.1, near the bottom of image). Blue circles, miRNAs; orange circles, IncRNAs; green circles, mRNAs. 
Table I. Significant differentially expressed lncRNAs for ceRNA network construction (fold change $>2.0, \mathrm{P}<0.05$ ).

\begin{tabular}{llcrc}
\hline IncRNAs & GeneSymbol & P-value & Fold change & Regulation \\
\hline T162623 & G037602 & 0.0002817 & 5.1090401 & Down \\
T347175 & G081784 & 0.0013410 & 2.4223804 & Down \\
uc001kfc. 1 & AK130076 & 0.0077363 & 13.0961017 & Down \\
T044224 & G010228 & 0.0101259 & 2.5568534 & Down \\
ENST00000420253 & AC093495.4 & 0.0006598 & 5.4432596 & Up \\
\hline
\end{tabular}

A

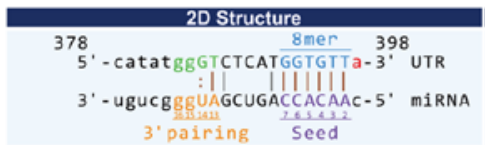

B

B Regulation of ion transport Cell communication involved in cardiac conduction Regulation of transporter activity Regulation of cation channel activity Cardiac conduction

Regulation of transmembrane transporter activity Regulation of ion transmembrane transporter activity Cell communication by electrical coupling Cell communication by electrical coupling involved in cardiac conduction
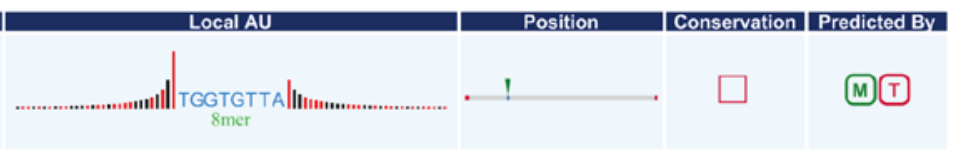

C

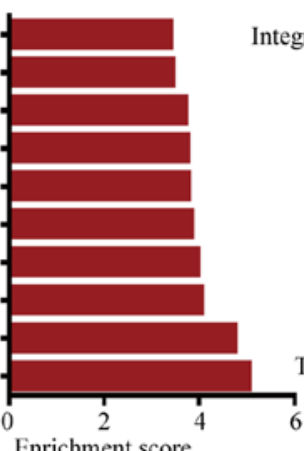

Integral component of plasma membrane Nuclear heterochromatin Plasma membrane protein complex Spindle midzone Golgi cisterna membrane Membrane protein complex Golgi stack Ion channel complex Transporter complex Transmembrane transporter complex

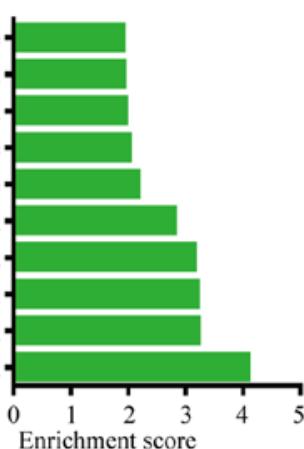

D

Estrogen receptor binding Ubiquitin-like protein conjugating enzyme binding Substrate-specific channel activity Ubiquitin conjugating enzyme binding Gated channel activity Ion channel activity Ligand-gated ion channel activity Ligand-gated channel activity Cation channel activity Protein kinase A regulatory subunit binding

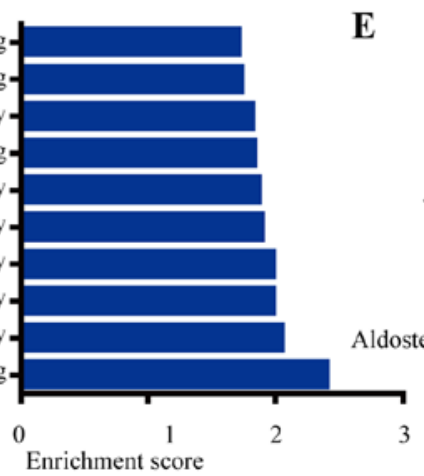

E

Salivary secretion Oxytocin signaling pathway mTOR signaling pathway • Morphine addiction Jak-STAT signaling pathway Gastric acid secretion Dilated cardiomyopathy cAMP signaling pathway e synthesis and secretion Adrenergic signaling in cardiomyocytes

$$
\begin{aligned}
& \begin{array}{llll}
1.75 & 2.00 & 2.25 & 2.50 \\
\text { Enrichment score } &
\end{array} \\
& \text { P-value Selection counts } \\
& \begin{array}{l}
0.020: 3.0 \\
0.005
\end{array}:^{4.0}
\end{aligned}
$$

Figure 7. 2D structure of miRNA vs. lncRNA and enriched functional terms in the lncRNA/miRNA/mRNA ceRNA network. (A) The 2D structure of the miR-21-3p vs. uc001kfc.1 (AK130076). The 2D structure revealed the specific location of the binding sites on the full-length sequence of lncRNA. The type of binding site is 8mer, indicating perfectly matched positions 2 to 8 of the mature miRNA (the seed + position 8 ) followed by an 'A'; ' ' indicates an exact match; ' $:$ ' indicates G:U pairing. Local AU indicates accessibility of binding sites; the red bar indicates that the location is A:U; the darker red bar indicates higher weighing of A:U. Position: Relative position of binding sites on the lncRNA. Conservation: Relative conservation of the seed complementary region between species. However, there is insufficient data for constructing a phylogenetic tree of different species for lncRNAs. (B-D) The top-10 significantly enriched GO terms in the lncRNA/miRNA/mRNA ceRNA network of cholesteatoma $(\mathrm{P}<0.05)$. B, biological process; C, cellular component; and $\mathrm{D}$, molecular function. Enrichment score indicates the enrichment score value of the GO ID, equivalent to $-\log _{10}$ (P-value). (E) The top-10 significantly enriched KEGG terms in IncRNA/miRNA/mRNA ceRNA network of cholesteatoma $(\mathrm{P}<0.05)$. 'P-value' indicates the enrichment $\mathrm{P}$-value of the Pathway ID using Fisher's exact test. 'Selection counts' indicates the Count of the differentially expressed gene entities directly associated with the listed Pathway ID. 'Enrichment score' indicates the enrichment score value of the Pathway ID, equivalent to '- $\log _{10}$ (P-value)'.

network, we observed that lncRNA-uc001kfc.1 interacted with miR-21-3p (Figs. 6 and 7A), a microRNA belonging to the miR-21 family, which promotes the formation and invasion of cholesteatoma (7,8). (To make the IncRNA-uc001kfc.1-mediated ceRNA network easier to identify, we separated it from the original ceRNA network). The 2D structure of the miR-21-3p on uc001kfc. 1 indicated that the binding site is 8 mer, perfectly matching positions 2 to 8 of the mature miR-21-3p (Fig. 7A).

Furthermore, to better explore the ceRNA potential of lncRNAs in cholesteatoma, we performed GO and KEGG pathway analysis for the lncRNA/miRNA/mRNA network. In GO analysis, gene product enrichment in $\mathrm{BP}, \mathrm{MF}$, and $\mathrm{CC}$ 
were analyzed $(\mathrm{P}<0.05)$ and the top-10 enriched GO terms of the three aspects are listed in Fig. 7B-D. GO analysis revealed that protein kinase activator activity (GO:0030295) as well as protein kinase A binding (GO:0051018) and protein kinase A regulatory subunit binding (GO:0034237) were the most significant molecular functions displayed in the ceRNA network. Protein kinase can regulate protein phosphorylation, which is involved in many aspects of cell biology and contributes to cholesteatoma formation (27). In KEGG pathway analysis, as depicted in Fig. 7E, the cAMP signaling pathway, the JAK/STAT signaling pathway, and the mTOR signaling pathway were related to cell migration, cell cycle, and survival (http://www.genome.jp/kegg/). Moreover, JAK/STAT signaling was also involved in the molecular mechanisms underlying cholesteatoma (28).

\section{Discussion}

The present study provides a comprehensive analysis of lncRNA and mRNA expression profiles in cholesteatoma and matched normal skin tissues by microarray analysis. Microarray data indicated that both lncRNA and mRNA profiles were distinctly different between cholesteatoma and normal skin specimens. In cholesteatoma tissues, a total of 181 upregulated lncRNAs and 606 downregulated lncRNAs were differentially expressed compared with normal skin tissues, which indicated that lncRNAs may play important roles in cholesteatoma. Among them, intergenic lncRNAs account for the largest category (69.47\%). This is meaningful, since intergenic lncRNAs are a type of IncRNA that are transcribed and act as cis-regulators when close to protein-coding genes during gene translation, which makes them the best candidates for in-depth study of transcriptional regulation of neighboring genes (14). To verify the reliability of our microarray data, 3 upregulated and 3 downregulated lncRNAs were selected to conduct qRT-PCR in a total of 7 pairs of samples. The results of qRT-PCR were consistent with the microarray assays, which demonstrated that the microarray data were highly reliable.

Since lncRNAs can regulate the expression of neighboring and overlapping coding genes, GO enrichment analysis of differentially expressed mRNAs can display the functional roles of IncRNAs. Based on differentially expressed mRNAs data from cholesteatoma specimens, we identified the framework of the gene functions and analyzed the gene product activities in terms of BP, MF, and CC by GO enrichment analysis. In $\mathrm{BP}$ analysis of upregulated genes, we observed that regulation of phosphatidylinositol 3-kinase signaling (GO:0014066), phosphatidylinositol 3-kinase signaling (GO:0014065), and positive regulation of phosphatidylinositol 3-kinase signaling (GO:0014068) were three of the most significant processes. Furthermore, in MF analysis of upregulated genes, phosphatidylinositol 3-kinase binding (GO:0043548) was the most significant gene activity process. All the gene activity processes aforementioned are all related to upregulation of phosphatidylinositol 3-kinase (PI3K) signaling in cholesteatoma tissues. PI3Ks are enzymes that catalyze the phosphorylation of phosphatidylinositol (PtdIns). PI3K signaling has been reported to function in metabolic control, immunity, angiogenesis, and cardiovascular homeostasis (29).
Furthermore, the PI3K pathway is also a critical regulator of cell survival and proliferation (30). During the past years, PI3K signaling activity has been reported to promote the genesis of cholesteatoma. A previous study reported that epithelial keratinocytes in cholesteatoma are protected against programmed cell death by activation of the PI3K/Akt signaling pathway (31). Akt, also known as serine kinase PKB, is a downstream effector of PI3K (29). In addition, Yune and Byun discovered that cellular survival mechanisms are related to cholesteatoma epithelial hyper-proliferation via reduced PTEN and increased PI3K/Akt signaling pathway activation (32). KEGG pathway analysis for differentially expressed mRNAs revealed that 7 upregulated pathways and 10 downregulated pathways could participate in the mechanisms underlying cholesteatoma. Bacterial invasion of epithelial cells is one of the upregulated pathways. Our results support a previous study in which it was revealed that bacterial infection may promote the enlargement of cholesteatoma and destruction of local structures (33). Therefore, functional analysis implied that IncRNAs may play important roles in cholesteatoma pathogenesis by regulating the neighboring and overlapping coding genes.

During the past decade, though many studies have reported that lncRNAs are involved in the epigenetic mechanisms of many diseases, the exact regulatory pathways of most lncRNAs with other transcripts are still largely unknown. In the present study, we constructed an lncRNA/miRNA/mRNA network to discover the relationship between IncRNAs, miRNAs, and mRNAs. The ceRNA hypothesis proposes that, by shared MREs, IncRNAs can sequester the miRNA activity, thereby upregulating the targeted mRNAs (12). In the present study, in the lncRNA/miRNA/mRNA network,

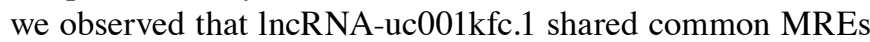
with hsa-miR-21-3p. Compared with normal skin tissues, IncRNA-uc001kfc.1 was confirmed to have low expression in cholesteatoma by both microarray analysis and qRT-PCR. Previous studies reported that hsa-miR-21 was overexpressed in cholesteatoma; and when hsa-miR-21 is upregulated, the number of proliferative as well as migrated cholesteatoma keratinocytes increases significantly $(7,8,34)$. In the present study, IncRNA-uc001kfc.1 was significantly downregulated. Therefore, we presume that $\operatorname{lncRNA}$-uc $001 \mathrm{kfc} .1$ may play a key role in cholesteatoma pathogenesis and function as an 'endogenous sponge' for miR-21-3p; and when lncRNA-uc $001 \mathrm{kfc} .1$ is downregulated in cholesteatoma, hsa-miR-21-3p becomes transcriptionally active, regulating relative genes, and thus resulting in the hyper-proliferation and migration of keratinocytes. This hypothesis gives us hope that lncRNA-uc001kfc. 1 mimics may be a potential drug treatment for cholesteatoma. In addition, more functional experiments are required to validate the hypothesis.

To further explore the ceRNA potential of lncRNAs in cholesteatoma, GO and KEGG pathway analyses were performed to analyze the functions of IncRNA-related genes in the lncRNA/miRNA/mRNA network. In MF fold enrichment of GO analysis, protein kinase activator activity (GO:0030295) was one of the most significantly enriched processes, which represents 'Binds to and increases the activity of a protein kinase, an enzyme that phosphorylates a protein' (http://www.geneontology.org). Protein phosphorylation is 
important in the control of cell metabolism (35). Increased phosphorylated Akt (p-Akt) expression can induce cell hyper-proliferation in cholesteatoma by activating the PI3K/Akt pathway (32). In another study, phosphorylation of HSP27, which is triggered by the Ras/Raf/ERK1/2 and MAPK pathways, was involved in the activation of epithelial cell migration, angiogenesis, and proliferation, subsequently resulting in the growth of cholesteatoma (36). Additionally, in the JAK/STAT signaling pathway, the phosphorylation of STAT3 can promote cholesteatoma epithelial hyper-proliferation by cell cycle acceleration, cellular differentiation promotion, and inhibition of apoptosis (28). Among the most significant KEGG pathways of the network, the JAK/STAT signaling pathway, just as depicted above, has been previously reported to be involved in the pathogenesis of cholesteatoma (28) and our study further supported these findings. Moreover, the cAMP signaling pathway, the JAK/STAT signaling pathway, and the mTOR signaling pathway, which are associated with the PI3K/Akt signaling pathway, may contribute to cholesteatoma formation through their involvement in cell cycle, migration, and survival (http://www.genome.jp/kegg/). All functional analysis of the lncRNA/miRNA/mRNA network further elucidated that lncRNAs had ceRNA potential and may play key roles in cholesteatoma pathogenesis.

In summary, this is the first study to examine lncRNA expression profiles in cholesteatoma using a microarray analysis and we discovered that lncRNA expression patterns were significantly altered in cholesteatoma. In addition, by constructing an lncRNA/miRNA/mRNA ceRNA network, we found that lncRNAs may function as ceRNAs during cholesteatoma formation. Our findings shed novel light on the pathogenesis of cholesteatoma and provide potential therapeutic targets for the treatment of cholesteatoma. In subsequent research, we will focus on the functional studies of lncRNAs, such as gain-of-function or loss-of-function of IncRNA-uc001kfc.1, to further explore the precise molecular mechanisms of cholesteatoma.

\section{Acknowledgments}

Not applicable.

\section{Funding}

This study was supported by grants from the National Natural Science Foundation of China (no. 81470698), the Beijing Natural Science Foundation (no. 7172176) and the Graduate Innovation Foundation of Peking Union Medical College (no. 1002-02-01).

\section{Availability of data and materials}

The datasets used during the present study are available from the corresponding author upon reasonable request.

\section{Authors' contributions}

HY and JJG conceived and designed the study. JJG, QT, XHZ, SHW, YLZ, WBL performed the experiments. JJG wrote the paper. ZQG, SHW and HY reviewed and edited the manuscript.
All authors read and approved the manuscript and agree to be accountable for all aspects of the research in ensuring that the accuracy or integrity of any part of the work are appropriately investigated and resolved.

\section{Ethics approval and consent to participate}

All experimental protocols were approved by the Ethics Committee of Peking Union Medical College Hospital (Beijing, China).

\section{Consent for publication}

Not applicable.

\section{Competing interests}

The authors declare that they have no competing interests.

\section{References}

1. Louw L: Acquired cholesteatoma pathogenesis: Stepwise explanations. J Laryngol Otol 124: 587-593, 2010.

2. Kuo CL, Shiao AS, Yung M, Sakagami M, Sudhoff H, Wang CH, Hsu CH and Lien CF: Updates and knowledge gaps in cholesteatoma research. Biomed Res Int 2015: 854024, 2015.

3. Tono T, Sakagami M, Kojima H, Yamamoto Y, Matsuda K, Komori M, Hato N, Morita Y and Hashimoto S: Staging and classification criteria for middle ear cholesteatoma proposed by the Japan Otological Society. Auris Nasus Larynx 44: 135-140, 2017.

4. Crowson MG, Ramprasad VH, Chapurin N, Cunningham CD III and Kaylie DM: Cost analysis and outcomes of a second-look tympanoplasty-mastoidectomy strategy for cholesteatoma. Laryngoscope 126: 2574-2579, 2016.

5. Prasad SC, Piras G, Piccirillo E, Taibah A, Russo A, He J and Sanna M: Surgical strategy and facial nerve outcomes in petrous bone cholesteatoma. Audiol Neurootol 21: 275-285, 2016.

6. Maniu A, Harabagiu O, Perde Schrepler M, Cătană A, Fănuţă B and Mogoantă CA: Molecular biology of cholesteatoma. Rom J Morphol Embryol 55: 7-13, 2014.

7. Friedland DR, Eernisse R, Erbe C, Gupta N and Cioffi JA: Cholesteatoma growth and proliferation: Posttranscriptional regulation by microRNA-21. Otol Neurotol 30: 998-1005, 2009.

8. Chen X and Qin Z: Post-transcriptional regulation by microrna-21 and let-7a microRNA in paediatric cholesteatoma. J Int Med Res 39: 2110-2118, 2011.

9. Li N and Qin ZB: Inflammation-induced miR-802 promotes cell proliferation in cholesteatoma. Biotechnol Lett 36: 1753-1759, 2014.

10. Zhang W, Chen X and Qin Z: MicroRNA let-7a suppresses the growth and invasion of cholesteatoma keratinocytes. Mol Med Rep 11: 2097-2103, 2015.

11. Bartel DP: MicroRNAs: Target recognition and regulatory functions. Cell 136: 215-233, 2009.

12. Salmena L, Poliseno L, Tay Y, Kats L and Pandolfi PP: A ceRNA hypothesis: The Rosetta Stone of a hidden RNA language? Cell 146: 353-358, 2011.

13. Bak RO and Mikkelsen JG: miRNA sponges: Soaking up miRNAs for regulation of gene expression. Wiley Interdiscip Rev RNA 5: 317-333, 2014.

14. Ponting CP, Oliver PL and Reik W: Evolution and functions of long noncoding RNAs. Cell 136: 629-641, 2009.

15. Chen LL and Carmichael GG: Decoding the function of nuclear long non-coding RNAs. Curr Opin Cell Biol 22: 357-364, 2010.

16. Rogoyski OM, Pueyo JI, Couso JP and Newbury SF: Functions of long non-coding RNAs in human disease and their conservation in Drosophila development. Biochem Soc Trans 45: 895-904, 2017.

17. Santoro M, Nociti V, Lucchini M, De Fino C, Losavio FA and Mirabella M: Expression profile of long non-coding RNAs in serum of patients with multiple sclerosis. J Mol Neurosci 59: 18-23, 2016. 
18. Elia L and Quintavalle M: Epigenetics and vascular diseases: Influence of non-coding RNAs and their clinical implications. Front Cardiovasc Med 4: 26, 2017.

19. Zhang X,Liu G, Qiu J,Zhang N, Ding J and Hua K: E2F1-regulated long non-coding RNA RAD51-AS1 promotes cell cycle progression, inhibits apoptosis and predicts poor prognosis in epithelial ovarian cancer. Sci Rep 7: 4469, 2017.

20. Xu J, Qian Y, Ye M, Fu Z, Jia X, Li W, Xu P, Lv M, Huang L, Wang L, et al: Distinct expression profile of lncRNA in endometrial carcinoma. Oncol Rep 36: 3405-3412, 2016.

21. Pan B, Zhou HX, Liu Y, Yan JY, Wang Y, Yao X, Deng YQ Chen SY, Lu L, Wei ZJ, et al: Time-dependent differential expression of long non-coding RNAs following peripheral nerve injury. Int J Mol Med 39: 1381-1392, 2017.

22. Jiao C, Song Z, Chen J, Zhong J, Cai W, Tian S, Chen S, Yi Y and Xiao Y: IncRNA-UCA1 enhances cell proliferation through functioning as a ceRNA of Sox4 in esophageal cancer. Oncol Rep 36: 2960-2966, 2016.

23. Sui J, Li YH, Zhang YQ, Li CY, Shen X, Yao WZ, Peng H, Hong WW, Yin LH, Pu YP and Liang GY: Integrated analysis of long non-coding RNAassociated ceRNA network reveals potential lncRNA biomarkers in human lung adenocarcinoma. Int J Oncol 49: 2023-2036, 2016

24. Liu W, Ren H, Ren J, Yin T, Hu B, Xie S, Dai Y, Wu W, Xiao Z, Yang $X$ and Xie D: The role of EGFR/PI3K/Akt/cyclinD1 signaling pathway in acquired middle ear cholesteatoma. Mediators Inflamm 2013: 651207, 2013.

25. Ergun S, Zheng X and Carlsoo B: Expression of transforming growth factor-alpha and epidermal growth factor receptor in middle ear cholesteatoma. Am J Otol 17: 393-396, 1996.

26. Likus W, Siemianowicz K, Markowski J, Wiaderkiewicz J, Kostrząb-Zdebel A, Jura-Szołtys E, Dziubdziela W, Wiaderkiewicz R and Łos MJ: Bacterial infections and osteoclastogenesis regulators in men and women with cholesteatoma. Arch Immunol Ther Exp 64: 241-247, 2016.

27. Huisman MA, De Heer E and Grote JJ: Terminal differentiation and mitogen-activated protein kinase signaling in human cholesteatoma epithelium. Otol Neurotol 27: 422-426, 2006.
28. Liu W, Xie S, Chen X, Rao X, Ren H, Hu B, Yin T, Xiang Y and Ren J: Activation of the IL-6/JAK/STAT3 signaling pathway in human middle ear cholesteatoma epithelium. Int J Clin Exp Pathol 7: 709-715, 2014.

29. Hawkins PT, Anderson KE, Davidson K and Stephens LR: Signalling through Class I PI3Ks in mammalian cells. Biochem Soc Trans 34: 647-662, 2006.

30. Song G, Ouyang G and Bao S: The activation of Akt/PKB signaling pathway and cell survival. J Cell Mol Med 9: 59-71, 2005.

31. Huisman MA, De Heer E and Grote JJ: Survival signaling and terminal differentiation in cholesteatoma epithelium. Acta Otolaryngol 127: 424-429, 2007.

32. Yune TY and Byun JY: Expression of PTEN and phosphorylated Akt in human cholesteatoma epithelium. Acta Otolaryngol 129: 501-506, 2009.

33. Brook I and Burke P: The management of acute, serous and chronic otitis media: The role of anaerobic bacteria. J Hosp Infect 22 (Suppl A): S75-S87, 1992.

34. Chen X, Li X and Qin Z: MicroRNA-21 promotes the proliferation and invasion of cholesteatoma keratinocytes. Acta Otolaryngol 136: 1261-1266, 2016.

35. Humphrey SJ, James DE and Mann M: Protein phosphorylation: A Major switch mechanism for metabolic regulation. Trends Endocrinol Metab 26: 676-687, 2015.

36. Ho KY, Yeh TS, Huang HH, Hung KF, Chai CY, Chen WT, Tsai SM, Chang NC, Chien CY, Wang HM and Wu YJ: Upregulation of phosphorylated HSP27, PRDX2, GRP75, GRP78 and GRP94 in acquired middle ear cholesteatoma growth. Int J Mol Sci 14: 14439-14459, 2013.

This work is licensed under a Creative Commons Attribution-NonCommercial-NoDerivatives 4.0 International (CC BY-NC-ND 4.0) License. 